\title{
Totally Real Statistical Submanifolds
}

\author{
Mirjana MILIJEVIĆ* \\ Department of Mathematics, Hokkaido University, Sapporo 060-0810, Japan
}

Received November 20, 2014; final version accepted January 14, 2015

\begin{abstract}
We prove that a semi-parallel totally real statistical submanifold with some natural conditions is totally geodesic if it is of non zero constant curvature, which is corresponding to the Kassabov theorem in the submanifold theory of Kähler manifolds. Moreover, we construct four dimensional holomorphic statistical manifolds using $g$-natural metrics (cf. [1]).
\end{abstract}

KEYWORDS: Holomorphic statistical manifolds, totally real submanifolds, semi-parallel submanifolds

\section{Introduction}

The investigation of totally real submanifolds of Kähler manifolds has a long history. The purpose of this paper is to give fundamental properties of totally real submanifolds of holomorphic statistical manifolds, which are new objects originating from information geometry.

A submanifold $M$ of a Kähler manifold $\bar{M}$ is totally real if the image $J T_{x} M$ of the tangent space $T_{x} M$ under the almost complex structure $J$ is a subspace of the normal space $T_{x}^{\perp} M$ for each point $x$ in $M$. Let $f$ be the endomorphism such that $f V$ denotes the normal component of the vector field $J V$ for a normal vector field $V$. The map $f$ defines an $f$-structure in the normal bundle, and it is said to be parallel if the condition $D^{0} f=0$, i.e. $D_{X}^{0}(f V)-f D_{X}^{0} V=0$, for $X \in \Gamma(T M)$ and $V \in \Gamma\left(T^{\perp} M\right)$, is satisfied. With $D^{0}$ we denoted the normal connection, i.e. the normal part of $\bar{\nabla}_{X}^{0} V$, where $\bar{\nabla}^{0}$ is the Levi-Civita connection. A submanifold $M$ is said to have parallel second fundamental form $h^{0}$, if

$$
\left(\widetilde{\nabla}_{X}^{0} h^{0}\right)(Y, Z):=D_{X}^{0} h^{0}(Y, Z)-h^{0}\left(\nabla_{X}^{0} Y, Z\right)-h^{0}\left(Y, \nabla_{X}^{0} Z\right)
$$

equals zero. $\widetilde{\nabla}^{0}$ is known as the connection of van der Waerden-Bortolotti. Here, $\nabla^{0}$ denotes the induced connection from the connection $\bar{\nabla}^{0}$. More general class of submanifolds are semi-parallel, i.e. submanifolds satisfying

$$
\left(R^{\widetilde{\nabla}^{0}}(X, Y) h^{0}\right)(Z, W):=\left(\widetilde{\nabla}_{X}^{0}\left(\widetilde{\nabla}_{Y}^{0} h^{0}\right)\right)(Z, W)-\left(\widetilde{\nabla}_{Y}^{0}\left(\widetilde{\nabla}_{X}^{0} h^{0}\right)\right)(Z, W)-\left(\widetilde{\nabla}_{[X, Y]}^{0} h^{0}\right)(Z, W),
$$

equals zero. These submanifolds are studied by Kassabov in 1986, under the condition that $f$ is parallel in the normal bundle (cf. [4]). Kassabov proved that totally real semi-parallel submanifolds of constant curvature are totally geodesic or flat.

A statistical manifold is a triple $(\bar{M}, \bar{\nabla}, \bar{g})$, where $\bar{\nabla}$ is a connection of torsion free, and $\bar{g}$ is a Riemannian metric satisfying the Codazzi equation (see Definition 2.1). Furthermore, a quadruple $(\bar{M}, \bar{\nabla}, \bar{g}, J)$ is called a holomorphic statistical manifold if the triple $(\bar{M}, \bar{\nabla}, \bar{g})$ is a statistical manifold and the Kähler form for $\bar{g}$ and $J$ is $\bar{\nabla}$-parallel (see Definition 4.1). On a statistical manifold the dual connection $\bar{\nabla}^{*}$ of $\bar{\nabla}$ with respect to $\bar{g}$ is naturally obtained by $X \bar{g}(Y, Z)=\bar{g}\left(\bar{\nabla}_{X} Y, Z\right)+\bar{g}\left(Y, \bar{\nabla}_{X}^{*} Z\right)$. Accordingly, for submanifolds in statistical manifolds we have pairs of induced connections $\nabla, \nabla^{*}$, second fundamental forms $h, h^{*}$, shape operators $A, A^{*}$, and normal connections $D, D^{*}$ satisfying equations analogous to the Gauss and the Weingarten ones for $\bar{\nabla}$ and $\bar{\nabla}^{*}$, respectively, though the induced metric $g$ is unique.

In this paper we are interested in totally real submanifolds in holomorphic statistical manifolds. Naturally, we want to see whether the results in the classical submanifold theory, for example Kassabov's theorems, hold for our setting or not. It appears that, when the ambient manifold is holomorphic statistical the condition that $f$ is parallel in the normal bundle with respect to $\bar{\nabla}$ or $\bar{\nabla}^{*}$ could not give the expected results, without any additional assumptions. Therefore, we needed to find the corresponding condition in our setting to the condition $D^{0} f=0$ in Kähler manifolds. Since in Lagrangian submanifolds (see Remark 3.5), for the normal connections $D$ and $D^{*}$ the condition $D_{X}(f V)=f D_{X}^{*} V$ is satisfied for $X \in \Gamma(T M)$ and $V \in \Gamma\left(T^{\perp} M\right)$, we generalize and examine it on any totally real submanifold. Besides, when the holomorphic structure is trivial this condition is equivalent to $D^{0} f=0$. We study semi-parallel submanifolds with respect to the connection $\bar{\nabla}$, and the main result is given as follows. 
Theorem 1.1. Let $(\bar{M}, \bar{\nabla}, \bar{g}, J)$ be a holomorphic statistical manifold and $M$ a totally real submanifold of $\bar{M}$. Suppose: 1) $D_{X}(f V)=f D_{X}^{*} V$,

2) $(\nabla, g)$ is of constant curvature $c \neq 0$.

If $M$ is semi-parallel for $\bar{\nabla}$, then $M$ is totally geodesic for $\bar{\nabla}$.

Corollary 1.2. Let $(\bar{M}, \bar{\nabla}, \bar{g}, J)$ be a holomorphic statistical manifold and $M$ a Lagrangian submanifold of $\bar{M}$. If $(\nabla, g)$ is of constant curvature $c \neq 0$ and $M$ is semi-parallel for $\bar{\nabla}$, then $M$ is totally geodesic for $\bar{\nabla}$.

We remark that the dual assertion of Theorem 1.1 holds. Namely, if $M$ is semi-parallel for $\bar{\nabla}^{*}$, then $M$ is totally geodesic for $\bar{\nabla}^{*}$.

This paper is organized as follows. In $\S 2$ we remind the definition of statistical manifolds and fundamental equations for statistical submanifolds. In $\S 3$ we review the fundamental properties of CR submanifolds of Kähler manifolds. We define totally real submanifolds as a special class of CR submanifolds. In $\S 4$ we give a definition of holomorphic statistical manifolds. We get the results for submanifolds in holomorphic statistical manifolds, similar to the ones of Yano and Kon in Kähler manifolds ([7]). In $\$ 5$ we give the proof of Theorem 1.1. In $\S 6$ we construct four dimensional holomorphic statistical structures, using a $g$-natural metric constructed by Oproiu in 1999 ([5]). These structures depend on nine functions. By choosing these functions adequately, we can obtain holomorphic statistical manifolds with desired properties. This correspondence will be matter of further studies.

\section{Statistical Submanifolds}

Throughout this paper, let $\bar{M}$ be a $C^{\infty}$ manifold of dimension $\bar{m} \geq 2, \bar{\nabla}$ an affine connection on $\bar{M}$, and $\bar{g}$ a Riemannian metric on $\bar{M}$. We denote by $\Gamma(E)$ the set of all the $C^{\infty}$ sections of a vector bundle $E \rightarrow \bar{M}$.

Definition 2.1. 1) $(\bar{M}, \bar{\nabla}, \bar{g})$ is called a statistical manifold if

(i) $\bar{\nabla}$ is of torsion free and

(ii) $\left(\bar{\nabla}_{X} \bar{g}\right)(Y, Z)=\left(\bar{\nabla}_{Y} \bar{g}\right)(X, Z)$ for $X, Y, Z \in \Gamma(T \bar{M})$.

2) $\bar{\nabla}^{*}$ is called the dual connection of $\bar{\nabla}$ with respect to $\bar{g}$ if

$$
X \bar{g}(Y, Z)=\bar{g}\left(\bar{\nabla}_{X} Y, Z\right)+\bar{g}\left(Y, \bar{\nabla}_{X}^{*} Z\right), \quad X, Y, Z \in \Gamma(T \bar{M}) .
$$

We remark that if $(\bar{M}, \bar{\nabla}, \bar{g})$ is a statistical manifold, so is $\left(\bar{M}, \bar{\nabla}^{*}, \bar{g}\right)$.

Definition 2.2. A statistical manifold $(\bar{M}, \bar{\nabla}, \bar{g})$ is said to be of constant curvature $c \in \mathbb{R}$ if

$$
R^{\bar{\nabla}}(X, Y) Z=c\{\bar{g}(Y, Z) X-\bar{g}(X, Z) Y\}, \quad X, Y, Z \in \Gamma(T \bar{M}),
$$

where $R^{\bar{\nabla}}(X, Y) Z:=\bar{\nabla}_{X} \bar{\nabla}_{Y} Z-\bar{\nabla}_{Y} \bar{\nabla}_{X} Z-\bar{\nabla}_{[X, Y]} Z$.

A statistical manifold $(\bar{M}, \bar{\nabla}, \bar{g})$ is of constant curvature $c$ if and only if $\left(\bar{M}, \bar{\nabla}^{*}, \bar{g}\right)$ is of constant curvature $c$. The fundamental equations for statistical submanifolds are given by P. W. Vos in 1989 (cf. [6]).

Definition 2.3. Let $(\bar{M}, \bar{\nabla}, \bar{g})$ be a statistical manifold, and $M$ a submanifold of $\bar{M}$. By $T_{x}^{\perp} M$ we denote the normal space of $M$, i.e. $T_{x}^{\perp} M:=\left\{v \in T_{x} \bar{M} \mid \bar{g}(v, w)=0, w \in T_{x} M\right\}$, and by $g$ the induced metric on $M$. We define

$$
\begin{aligned}
& \nabla, \nabla^{*}: \Gamma(T M) \times \Gamma(T M) \rightarrow \Gamma(T M), \\
& h, h^{*}: \Gamma(T M) \times \Gamma(T M) \rightarrow \Gamma\left(T^{\perp} M\right), \\
& A, A^{*}: \Gamma\left(T^{\perp} M\right) \times \Gamma(T M) \rightarrow \Gamma(T M),
\end{aligned}
$$

and

$$
D, D^{*}: \Gamma(T M) \times \Gamma\left(T^{\perp} M\right) \rightarrow \Gamma\left(T^{\perp} M\right)
$$

by

for $X, Y \in \Gamma(T M), V \in \Gamma\left(T^{\perp} M\right)$.

$$
\begin{aligned}
& \bar{\nabla}_{X} Y=\nabla_{X} Y+h(X, Y), \bar{\nabla}_{X} V=-A_{V} X+D_{X} V, \\
& \bar{\nabla}_{X}^{*} Y=\nabla_{X}^{*} Y+h^{*}(X, Y), \bar{\nabla}_{X}^{*} V=-A_{V}^{*} X+D_{X}^{*} V,
\end{aligned}
$$

Proposition 2.4. Let $M, g, \nabla, \nabla^{*}, h, h^{*}, A, A^{*}, D$ and $D^{*}$ be as in Definition 2.3. Then:

1) $(M, \nabla, g)$ and $\left(M, \nabla^{*}, g\right)$ are statistical manifolds, and $\nabla^{*}$ is dual of $\nabla$ with respect to $g$.

2) $D$ and $D^{*}$ are connections of $T^{\perp} M \rightarrow M$. Denoting by $g^{\perp}$ the metric of $T^{\perp} M$ induced from $\bar{g}$, we have

$$
X g^{\perp}(U, V)=g^{\perp}\left(D_{X} U, V\right)+g^{\perp}\left(U, D_{X}^{*} V\right), \quad U, V \in \Gamma\left(T^{\perp} M\right), \quad X \in \Gamma(T M) .
$$

3) $h, h^{*}, A$ and $A^{*}$ are tensor fields satisfying 


$$
\begin{aligned}
& h(X, Y)=h(Y, X), \quad h^{*}(X, Y)=h^{*}(Y, X), \\
& g\left(A_{V} X, Y\right)=\bar{g}\left(h^{*}(X, Y), V\right), \quad g\left(A_{V}^{*} X, Y\right)=\bar{g}(h(X, Y), V), \quad X, Y \in \Gamma(T M), \quad V \in \Gamma\left(T^{\perp} M\right) .
\end{aligned}
$$

Proposition 2.5. Let $M$ be a submanifold of $\bar{M}$. For simplicity, we denote by $\bar{R}:=R^{\bar{\nabla}}, \bar{R}^{*}:=R^{\bar{\nabla}^{*}}, R:=R^{\nabla}$, $R^{*}:=R^{\nabla^{*}}, R^{\perp}:=R^{D}$ and $R^{\perp *}:=R^{D^{*}}$ the curvature tensor fields of $\bar{\nabla}, \bar{\nabla}^{*}, \nabla, \nabla^{*}, D$ and $D^{*}$, respectively. For $X, Y, Z \in \Gamma(T M), V \in \Gamma\left(T^{\perp} M\right)$ we have

$$
\begin{aligned}
& \{\bar{R}(X, Y) Z\}^{\top}=R(X, Y) Z-A_{h(Y, Z)} X+A_{h(X, Z)} Y, \\
& \{\bar{R}(X, Y) Z\}^{\perp}=\left(\widetilde{\nabla}_{X} h\right)(Y, Z)-\left(\widetilde{\nabla}_{Y} h\right)(X, Z), \\
& \{\bar{R}(X, Y) V\}^{\top}=\left(\widetilde{\nabla}_{Y} A\right)_{V} X-\left(\widetilde{\nabla}_{X} A\right)_{V} Y, \\
& \{\bar{R}(X, Y) V\}^{\perp}=R^{\perp}(X, Y) V+h\left(Y, A_{V} X\right)-h\left(X, A_{V} Y\right),
\end{aligned}
$$

and their duals, i.e.,

$$
\begin{aligned}
& \left\{\bar{R}^{*}(X, Y) Z\right\}^{\top}=R^{*}(X, Y) Z-A_{h^{*}(Y, Z)}^{*} X+A_{h^{*}(X, Z)}^{*} Y, \\
& \left\{\bar{R}^{*}(X, Y) Z\right\}^{\perp}=\left(\widetilde{\nabla}_{X}^{*} h^{*}\right)(Y, Z)-\left(\widetilde{\nabla}_{Y}^{*} h^{*}\right)(X, Z), \\
& \left\{\bar{R}^{*}(X, Y) V\right\}^{\top}=\left(\widetilde{\nabla}_{Y}^{*} A^{*}\right)_{V} X-\left(\widetilde{\nabla}_{X}^{*} A^{*}\right)_{V} Y, \\
& \left\{\bar{R}^{*}(X, Y) V\right\}^{\perp}=R^{\perp *}(X, Y) V+h^{*}\left(Y, A_{V}^{*} X\right)-h^{*}\left(X, A_{V}^{*} Y\right),
\end{aligned}
$$

where \{\}$^{\top}\left(\{\}^{\perp}\right)$ denotes the tangential (normal) part of \{\} for $M$, and

$$
\begin{aligned}
& \left(\widetilde{\nabla}_{X} h\right)(Y, Z):=D_{X} h(Y, Z)-h\left(\nabla_{X} Y, Z\right)-h\left(Y, \nabla_{X} Z\right), \\
& \left(\widetilde{\nabla}_{Y} A\right)_{V} X:=\nabla_{Y}\left(A_{V} X\right)-A_{D_{Y} V} X-A_{V} \nabla_{Y} X .
\end{aligned}
$$

Proof. We prove only (2.1) and (2.2). Formulas (2.3)-(2.8) are obtained in a similar way. For $X, Y \in \Gamma(T M)$ we have

$$
\begin{aligned}
\bar{R}(X, Y) Z= & \bar{\nabla}_{X} \bar{\nabla}_{Y} Z-\bar{\nabla}_{Y} \bar{\nabla}_{X} Z-\bar{\nabla}_{[X, Y]} Z \\
= & \bar{\nabla}_{X}\left(\nabla_{Y} Z+h(Y, Z)\right)-\bar{\nabla}_{Y}\left(\nabla_{X} Z+h(X, Z)\right)-\left(\nabla_{[X, Y]} Z+h([X, Y], Z)\right) \\
= & R(X, Y) Z+h\left(X, \nabla_{Y} Z\right)-h\left(Y, \nabla_{X} Z\right)-h([X, Y], Z)+\bar{\nabla}_{X} h(Y, Z)-\bar{\nabla}_{Y} h(X, Z) \\
= & R(X, Y) Z-A_{h(Y, Z)} X+A_{h(X, Z)} Y+h\left(X, \nabla_{Y} Z\right) \\
& -h\left(Y, \nabla_{X} Z\right)-h([X, Y], Z)+D_{X} h(Y, Z)-D_{Y} h(X, Z) .
\end{aligned}
$$

From this Eqs. (2.1) and (2.2) follow.

Remark 2.6. Equation $(2.1)^{*}$ is considered as the dual of (2.1). In the similar way, if it is clear, we omit the dual equation and put $*$ to the number of original equation.

\section{Review of CR Submanifolds}

In this section we give basic properties of CR submanifolds in Kähler manifolds.

Proposition 3.1 (cf. [8], p. 76). Let $M$ be a submanifold of a Kähler manifold $\bar{M}$. We define $P \in \Gamma(E n d T M)$, $F \in \Gamma\left((T M)^{*} \otimes T^{\perp} M\right), t \in \Gamma\left(\left(T^{\perp} M\right)^{*} \otimes T M\right), f \in \Gamma\left(\right.$ End $\left.T^{\perp} M\right)$ by

$$
J X=P X+F X, \quad X \in \Gamma(T M),
$$

and

$$
J V=t V+f V, \quad V \in \Gamma\left(T^{\perp} M\right) .
$$

Then

$$
\begin{aligned}
& \bar{g}(F X, V)=-g(X, t V), \\
& P^{2}=-1_{T M}-t F, F P+f F=0, \\
& P t+t f=0, f^{2}=-1_{T M}-F t .
\end{aligned}
$$

Definition 3.2. Let $M$ be a submanifold of a Kähler manifold $\bar{M}$.

1) We define

$$
\begin{aligned}
& \mathcal{D}_{x}:=T_{x} M \cap J_{x}\left(T_{x} M\right), x \in M, \\
& \mathcal{D}_{x}^{\perp}:=\left\{v \in T_{x} M \mid g(v, w)=0, w \in \mathscr{D}_{x}\right\}, \\
& N_{x}:=\left\{\xi \in T_{x}^{\perp} M \mid \bar{g}\left(\xi, J_{x} w\right)=0, w \in T_{x} M\right\} .
\end{aligned}
$$


2) $M$ is called a $C R$ submanifold of $\bar{M}$ if

(i) $\mathcal{D}$ defines a $C^{\infty}$ distribution on $M$,

(ii) $J_{x} \mathcal{D}_{x}^{\perp} \subset T_{x}^{\perp} M, x \in M$.

From Definition 3.2 it follows that the tangent space $T_{x} \bar{M}$ splits as:

$$
T_{x} \bar{M}=T_{x} M \oplus T_{x}^{\perp} M=\left(\mathcal{D}_{x} \oplus \mathcal{D}_{x}^{\perp}\right) \oplus\left(J D_{x}^{\perp} \oplus N_{x}\right) .
$$

Proposition 3.3 (cf. [8], p. 86). Let $M$ be a CR submanifold of a Kähler manifold $\bar{M}$. For $X \in \Gamma(T M)$ and $V \in$ $\Gamma\left(T^{\perp} M\right)$ we have:

(1) $X \in \Gamma(D)$ if and only if $F X=0 . X \in \Gamma\left(D^{\perp}\right)$ if and only if $P X=0$.

(2) $V \in \Gamma\left(J D^{\perp}\right)$ if and only if $f V=0 . V \in \Gamma(N)$ if and only if $t V=0$.

(3) $F P=0, f F=0, t f=0, P t=0$.

(4) $P^{3}+P=0, f^{3}+f=0$.

Definition 3.4. Let $M$ be a CR submanifold of a Kähler manifold $\bar{M} . M$ is called a totally real submanifold of $\bar{M}$ if $\operatorname{dim} \mathscr{D}_{x}=0, x \in M$. Equivalently, $M$ is a totally real submanifold of $\bar{M}$ if and only if $P=0$.

Remark 3.5. A totally real submanifold $M$ of a Kähler manifold $\bar{M}$, such that $\operatorname{dim} M=\frac{1}{2} \operatorname{dim} \bar{M}$, is said to be a Lagrangian submanifold. Then $\operatorname{dim} N_{x}=0$ for any $x$ in $M$, and $f=0$. In particular, the condition 1$) D_{X}(f V)=f D_{X}^{*} V$ of Theorem 1.1 holds.

\section{Totally Real Statistical Submanifolds}

Definition 4.1. Let $(\bar{M}, J, \bar{g})$ be a Kähler manifold and $\bar{\nabla}$ an affine connection of $\bar{M}$. $(\bar{M}, \bar{\nabla}, \bar{g}, J)$ is called a holomorphic statistical manifold if

1) $(\bar{M}, \bar{\nabla}, \bar{g})$ is a statistical manifold and

2) $\bar{\omega}:=\bar{g}\left({ }^{*}, J^{*}\right)$ is a $\bar{\nabla}$ - parallel 2 - form on $\bar{M}$.

Lemma 4.2 (cf. [3]). Let $(\bar{M}, \bar{g}, J)$ be a Kähler manifold. If we define a connection $\bar{\nabla}$ as $\bar{\nabla}:=\nabla^{\bar{g}}+K$, where $K$ is a $(1,2)$-tensor field satisfying the next three conditions:

$$
\begin{aligned}
& K_{X} Y=K_{Y} X, \\
& \bar{g}\left(K_{X} Y, Z\right)=\bar{g}\left(Y, K_{X} Z\right), \\
& K_{X} J Y=-J K_{X} Y,
\end{aligned}
$$

for $X, Y, Z \in \Gamma(T M)$, then $(\bar{M}, \bar{\nabla}, \bar{g}, J)$ is a holomorphic statistical manifold.

Example 4.3. Let $\mathbb{C}^{2}=\left(\mathbb{R}^{4}, g_{0}, J_{0}\right)$ be the complex Euclidean space, that is $g_{0}=\sum_{i=1}^{4} d x^{i} \otimes d x^{i}$ and $J_{0} \frac{\partial}{\partial x^{i}}=\frac{\partial}{\partial x^{i+2}}$, $i=1,2, \quad J_{0} \frac{\partial}{\partial x^{i}}=-\frac{\partial}{\partial x^{i-2}}, \quad i=3,4$. For functions $\alpha_{j}$ on $\mathbb{R}^{4}, j=1, \ldots, 8$, define a $(1,2)$-tensor field $K=$ $\sum_{i, j, l=1}^{4} k_{i j}^{l} \frac{\partial}{\partial x^{l}} \otimes d x^{i} \otimes d x^{j}$ on $\mathbb{C}^{2}$ as follows:

$$
\begin{aligned}
& k_{11}^{1}=\alpha_{1}, k_{13}^{3}=k_{31}^{3}=k_{33}^{1}=-\alpha_{1}, k_{11}^{2}=k_{12}^{1}=k_{21}^{1}=\alpha_{2}, \\
& k_{13}^{4}=k_{31}^{4}=k_{14}^{3}=k_{41}^{3}=k_{23}^{3}=k_{32}^{3}=k_{34}^{1}=k_{43}^{1}=k_{33}^{2}=-\alpha_{2}, \\
& k_{11}^{3}=k_{13}^{1}=k_{31}^{1}=\alpha_{3}, k_{33}^{3}=-\alpha_{3}, \\
& k_{11}^{4}=k_{14}^{1}=k_{41}^{1}=k_{13}^{2}=k_{31}^{2}=k_{12}^{3}=k_{21}^{3}=k_{23}^{1}=k_{32}^{1}=\alpha_{4}, \\
& k_{33}^{4}=k_{34}^{3}=k_{43}^{3}=-\alpha_{4}, k_{12}^{2}=k_{21}^{2}=k_{22}^{1}=\alpha_{5}, \\
& k_{14}^{4}=k_{41}^{4}=k_{44}^{1}=k_{23}^{4}=k_{32}^{4}=k_{34}^{2}=k_{43}^{2}=k_{24}^{3}=k_{42}^{3}=-\alpha_{5}, \\
& k_{22}^{2}=\alpha_{6}, k_{24}^{4}=k_{42}^{4}=k_{44}^{2}=-\alpha_{6}, k_{22}^{4}=k_{24}^{2}=k_{42}^{2}=\alpha_{7}, k_{44}^{4}=-\alpha_{7}, \\
& k_{12}^{4}=k_{21}^{4}=k_{14}^{2}=k_{41}^{2}=k_{24}^{1}=k_{42}^{1}=k_{23}^{2}=k_{32}^{2}=k_{22}^{3}=\alpha_{8}, \\
& k_{34}^{4}=k_{43}^{4}=k_{44}^{3}=-\alpha_{8} .
\end{aligned}
$$

Then $K$ satisfies the conditions in Lemma 4.2, and we get a holomorphic statistical manifold $\bar{M}=\left(\mathbb{R}^{4}, \bar{\nabla}:=\nabla^{g_{0}}+\right.$ $\left.K, g_{0}, J_{0}\right)$.

Lemma 4.4. Let $(\bar{M}, \bar{\nabla}, \bar{g}, J)$ be a holomorphic statistical manifold. Then $\bar{\nabla}_{X} J Y=J \bar{\nabla}_{X}^{*} Y$ for $X, Y \in \Gamma(T \bar{M})$, where $\bar{\nabla}^{*}$ is the dual connection of $\bar{\nabla}$ with respect to $\bar{g}$ (cf. Definition 2.1 (2)).

Proof. For $X, Y, Z \in \Gamma(T \bar{M})$ we have

$$
X \omega\left(Y, J^{-1} Z\right)=X \bar{g}(Y, Z)
$$

from which it follows 


$$
\left(\bar{\nabla}_{X} \omega\right)\left(Y, J^{-1} Z\right)+\omega\left(\bar{\nabla}_{X} Y, J^{-1} Z\right)+\omega\left(Y, \bar{\nabla}_{X}\left(J^{-1} Z\right)\right)=\bar{g}\left(\bar{\nabla}_{X} Y, Z\right)+\bar{g}\left(Y, \bar{\nabla}_{X}^{*} Z\right)
$$

that is

$$
0=\bar{g}\left(\bar{\nabla}_{X} Y, Z\right)+\bar{g}\left(Y, \bar{\nabla}_{X}^{*} Z\right)-\omega\left(\bar{\nabla}_{X} Y, J^{-1} Z\right)-\omega\left(Y, \bar{\nabla}_{X}\left(J^{-1} Z\right),\right.
$$

from which we conclude

$$
0=\bar{g}\left(Y, \bar{\nabla}_{X}^{*} Z\right)-\bar{g}\left(Y, J \bar{\nabla}_{X}\left(J^{-1} Z\right)\right),
$$

i.e.

$$
\bar{\nabla}_{X}^{*} Z=-J \bar{\nabla}_{X}(J Z)
$$

Remark 4.5. $(\bar{M}, \bar{\nabla}, \bar{g}, J)$ is a special Kähler manifold if $\bar{\nabla}$ is flat (for the definition of special Kähler manifolds and further reading we refer to [2]). To show this, for $X, Y, Z \in \Gamma(T \bar{M})$ we calculate

$$
\begin{aligned}
\bar{g}\left(X,\left(\bar{\nabla}_{Z} J\right) Y\right) & =\bar{g}\left(X, \bar{\nabla}_{Z}(J Y)\right)-\bar{g}\left(X, J \bar{\nabla}_{Z} Y\right)=\bar{g}\left(X, \bar{\nabla}_{Z}(J Y)\right)-\bar{g}\left(X, \bar{\nabla}_{Z}^{*}(J Y)\right) \\
& =Z \bar{g}(X, J Y)-\bar{g}\left(\bar{\nabla}_{Z}^{*} X, J Y\right)-\bar{g}\left(X, \bar{\nabla}_{Z}^{*}(J Y)\right)=\left(\bar{\nabla}_{Z}^{*} \bar{g}\right)(X, J Y),
\end{aligned}
$$

where we used Definition 2.1 ((2)). Putting $Y=J Y$ in (4.4), we get

$$
-\left(\bar{\nabla}_{Z}^{*} \bar{g}\right)(X, Y)=\bar{g}\left(X,\left(\bar{\nabla}_{Z} J\right) J Y\right) .
$$

Interchanging $Y$ and $Z$ in (4.5) and subtracting the obtained equation from (4.5), we get $\left(\bar{\nabla}_{Z} J\right) Y=\left(\bar{\nabla}_{Y} J\right) Z$, using Definition 2.1 (ii) and the properties of $J$.

Lemma 4.6. Let $(\bar{M}, \bar{\nabla}, \bar{g}, J)$ be a holomorphic statistical manifold and $M$ a submanifold of $\bar{M}$. Let $\nabla, \ldots, D^{*}$ be as in Definition 2.3, and $P, \ldots, f$ as in Proposition 3.1. For $X, Y \in \Gamma(T M), V \in \Gamma\left(T^{\perp} M\right)$ we have

$$
\begin{aligned}
& A_{F Y} X+t h^{*}(X, Y)=\nabla_{X}(P Y)-P \nabla_{X}^{*} Y, \\
& -h(X, P Y)+f h^{*}(X, Y)=D_{X}(F Y)-F \nabla_{X}^{*} Y, \\
& A_{f V} X-P A_{V}^{*} X=\nabla_{X}(t V)-t D_{X}^{*} V, \\
& -F A_{V}^{*} X-h(X, t V)=D_{X}(f V)-f D_{X}^{*} V,
\end{aligned}
$$

and their duals (4.6)*-(4.9)* hold (cf. Remark 2.6).

Proof. For $X, Y \in \Gamma(T M)$ we have

$$
\begin{aligned}
\bar{\nabla}_{X}(J Y) & =\bar{\nabla}_{X}(P Y)+\bar{\nabla}_{X}(F Y) \\
& =\nabla_{X}(P Y)-A_{F Y} X+h(X, P Y)+D_{X}(F Y)
\end{aligned}
$$

and

$$
\begin{aligned}
J \bar{\nabla}_{X}^{*} Y & =J\left(\nabla_{X}^{*} Y+h^{*}(X, Y)\right) \\
& =P \nabla_{X}^{*} Y+t h^{*}(X, Y)+F \nabla_{X}^{*} Y+f h^{*}(X, Y) .
\end{aligned}
$$

Subtracting (4.10) and (4.11) and using Lemma 4.4, we get (4.6) and (4.7). In a similar way we get the other formulas.

Proposition 4.7. Let $(\bar{M}, \bar{\nabla}, \bar{g}, J)$ be a holomorphic statistical manifold.

(1) Let $M$ be a totally real submanifold of $\bar{M}$. Then

$$
\begin{aligned}
& A_{J Y} X+t h^{*}(X, Y)=0, \\
& f h^{*}(X, Y)=D_{X}(J Y)-J \nabla_{X}^{*} Y, \\
& A_{f V} X=\nabla_{X}(t V)-t D_{X}^{*} V, \\
& -J A_{V}^{*} X-h(X, t V)=D_{X}(f V)-f D_{X}^{*} V,
\end{aligned}
$$

and their duals hold.

(2) Let $M$ be a totally real submanifold of $\bar{M}$ with $D_{X}(f V)=f D_{X}^{*} V$. Then

and their duals hold.

$$
\begin{aligned}
& A_{\xi}^{*}=0, \xi \in \Gamma(N), \\
& h(X, Y)=J A_{J Y}^{*} X, \\
& D_{X}(J Y)=J \nabla_{X}^{*} Y,
\end{aligned}
$$

Proof. (1) Formulas (4.12)-(4.15) follow directly from (4.6)-(4.9), since $P=0$.

(2) From (4.15) it follows that 


$$
-J A_{V}^{*} X=h(X, t V) \text {. }
$$

If $V=\xi \in \Gamma(N)$ then from (4.19) we conclude that $A_{\xi}^{*} X=0$, because of Proposition 3.3. If $V=J Y \in \Gamma\left(J D^{\perp}\right)$ then from (4.19) it follows $-J A_{J Y}^{*} X=h(X, t J Y)=h(X, t F Y)=-h(X, Y)$, because of (3.5). Hence we proved (4.16) and (4.17). To prove (4.18) we use (4.16)* and Proposition 2.4 (3), from which it follows that $h^{*}(X, Y) \in \Gamma\left(J D^{\perp}\right)$, i.e. $f h^{*}(X, Y)=0$ (Proposition $3.3(2)$ ). Now, from (4.13) follows (4.18).

Proposition 4.8. Let $(\bar{M}, \bar{\nabla}, \bar{g}, J)$ be a holomorphic statistical manifold. We define $I(X, Y):=\left(\bar{\nabla}_{X} J\right) Y$, $I^{*}(X, Y):=\left(\bar{\nabla}_{X}^{*} J\right) Y$ for $X, Y \in \Gamma(T \bar{M})$. Then $I(X, Y)=I(Y, X), I(X, Y)=-I^{*}(X, Y)$ and $I(X, J Y)=-J I(X, Y)$.

Proof. For $X, Y \in \Gamma(T \bar{M})$ we have

$$
I(X, Y)=\left(\bar{\nabla}_{X} J\right) Y=\bar{\nabla}_{X}(J Y)-J \bar{\nabla}_{X} Y=J \bar{\nabla}_{X}^{*} Y-J \bar{\nabla}_{X} Y .
$$

Interchanging $X$ and $Y$ in (4.20) we get

$$
I(Y, X)=J \bar{\nabla}_{Y}^{*} X-J \bar{\nabla}_{Y} X .
$$

Subtracting (4.20) and (4.21) we get $I(X, Y)-I(Y, X)=0$, since $\bar{\nabla}^{*}$ and $\bar{\nabla}$ are of torsion free. Next we calculate

$$
\begin{aligned}
& I(X, Y)=\left(\bar{\nabla}_{X} J\right) Y=\bar{\nabla}_{X}(J Y)-J \bar{\nabla}_{X} Y=J \bar{\nabla}_{X}^{*} Y-\bar{\nabla}_{X}^{*}(J Y)=-\left(\bar{\nabla}_{X}^{*} J\right) Y=-I^{*}(X, Y) . \\
& I(X, J Y)=\left(\bar{\nabla}_{X} J\right) J Y=\bar{\nabla}_{X}(J J Y)-J \bar{\nabla}_{X}(J Y)=-\left(\bar{\nabla}_{X} J\right) Y=-J I(X, Y) .
\end{aligned}
$$

Theorem 4.9. Let $(\bar{M}, \bar{\nabla}, \bar{g}, J)$ be a holomorphic statistical manifold and $M$ a totally real submanifold of $\bar{M}$ with $\operatorname{dim} \bar{M}=2 \operatorname{dim} M$. Then $I(X, Y) \in \Gamma\left(T^{\perp} M\right)$ for $X, Y \in \Gamma(T M)$ if and only if $h=h^{*}$, i.e. if and only if $A=A^{*}$.

Proof. For $X, Y \in \Gamma(T M)$ we calculate

$$
\begin{aligned}
\left(\bar{\nabla}_{X} J\right) Y= & \nabla_{X}(P Y)-P \nabla_{X} Y-A_{F Y} X-t h(X, Y) \\
& +D_{X}(F Y)-F \nabla_{X} Y+h(X, P Y)-f h(X, Y) .
\end{aligned}
$$

Comparing the tangential parts in (4.22) we have

$$
A_{F Y} X+\operatorname{th}(X, Y)=\nabla_{X}(P Y)-P \nabla_{X} Y .
$$

Since $P=0$, from (4.23) it follows

$$
A_{J Y} X=-\operatorname{th}(X, Y) \text {. }
$$

From (4.24) and (4.12) we get

$$
\operatorname{th}(X, Y)=t h^{*}(Y, X) .
$$

Example 4.10. Let $z=\left(z_{1}, z_{2}\right)$, with $z_{j}=x_{j}+\sqrt{-1} y_{j}, j=1,2$, denote the complex coordinates on $\mathbb{C}^{2}$. An immersion $\iota: \mathbb{R}^{2} \rightarrow \mathbb{C}^{2}$ given by $\iota:\left(x_{1}, x_{2}\right) \mapsto\left(x_{1}, x_{2}, 0,0\right)$ is totally real. Suppose $\alpha_{3}=\alpha_{4}=\alpha_{7}=\alpha_{8}=0$ for $\bar{M}=\left(\mathbb{R}^{4}, \bar{\nabla}:=\nabla^{g_{0}}+K, g_{0}, J_{0}\right)$ in Example 4.3. Then $I(X, Y)$ is orthogonal to $M$ for $X, Y \in \Gamma(T M)$.

\section{Semiparallel Statistical Submanifolds of Constant Curvature}

Definition 5.1. Let $M$ be a submanifold of a holomorphic statistical manifold $\bar{M}$.

(1) $M$ is said to be totally geodesic for the connection $\bar{\nabla}$ if $h=0$.

(2) $M$ is said to be totally geodesic for the connection $\bar{\nabla}^{*}$ if $h^{*}=0$.

Definition 5.2. Let $M$ be a submanifold of a holomorphic statistical manifold $\bar{M}$.

(1) $M$ is said to have parallel second fundamental form $h$ with respect to the connection $\bar{\nabla}$ if $\widetilde{\nabla} h=0$. Here,

$$
\left(\widetilde{\nabla}_{X} h\right)(Y, Z):=D_{X} h(Y, Z)-h\left(\nabla_{X} Y, Z\right)-h\left(Y, \nabla_{X} Z\right) .
$$

(2) $M$ is called a semi-parallel submanifold for the connection $\bar{\nabla}$ if $R^{\tilde{\nabla}}(X, Y) h=0$, where $\left(R^{\tilde{\nabla}}(X, Y) h\right)(Z, W):=$ $\left(\widetilde{\nabla}_{X}\left(\widetilde{\nabla}_{Y} h\right)\right)(Z, W)-\left(\widetilde{\nabla}_{Y}\left(\widetilde{\nabla}_{X} h\right)\right)(Z, W)-\left(\widetilde{\nabla}_{[X, Y]} h\right)(Z, W)$.

Proposition 5.3. Let $(\bar{M}, \bar{\nabla}, \bar{g}, J)$ be a holomorphic statistical manifold and $M$ a totally real submanifold of $\bar{M}$. Suppose:

1) $D_{X}(f V)=f D_{X}^{*} V$ for $X \in \Gamma(T M)$ and $V \in \Gamma\left(T^{\perp} M\right)$,

2) $(\nabla, g)$ is of constant curvature $c \neq 0$.

Then, for $Z \in \Gamma(T M)$, from $R^{\perp}(X, Y) J Z=0$ for $X, Y \in \Gamma(T M)$, it follows $Z=0$.

In particular, if $R^{\perp}(X, Y) H=0$ for $X, Y \in \Gamma(T M)$, then $H=0$. Here $H$ denotes the mean curvature vector field of $M$ with respect to $\bar{\nabla}$, that is $H=\frac{1}{m} t r_{g} h$.

Proof. For $X, Y, Z \in \Gamma(T M)$ we have 


$$
\begin{aligned}
0 & =R^{\perp}(X, Y) J Z=D_{X} D_{Y}(J Z)-D_{Y} D_{X}(J Z)-D_{[X, Y]}(J Z) \\
& =D_{X} J \nabla_{X}^{*} Z-D_{Y} J \nabla_{X}^{*} Z-J \nabla_{[X, Y]}^{*} Z \\
& =J R^{*}(X, Y) Z \\
& =c g(Y, Z) J X-\operatorname{cg}(X, Z) J Y .
\end{aligned}
$$

Putting $Y=Z$ and $X$ orthogonal to $Y$ in the last equation, we obtain $Z=0$. Next, using (4.17), we get $H=J\left(\frac{1}{m} \sum_{i=1}^{m} A_{J e_{i}}^{*} e_{i}\right)$, where $\left\{e_{i}\right\}_{i=1}^{m}$ is an orthonormal basis with respect to $g$. Therefore we conclude that from $R^{\perp}(X, Y) H=0$ it follows $H=0$.

The dual assertion of Proposition 5.3 holds, which means that $R^{\perp *}(X, Y) J Z=0$ implies $Z=0$ under the same assumption.

Proof of Theorem 1.1. For $X, Y \in \Gamma(T M)$ we have from $R^{\widetilde{\nabla}}(X, Y) h=0$,

$$
\begin{aligned}
m R^{\perp}(X, Y) H & =\sum_{i=1}^{m} R^{\perp}(X, Y) h\left(e_{i}, e_{i}\right)=2 \sum_{i=1}^{m} h\left(R(X, Y) e_{i}, e_{i}\right)=2 c \sum_{i=1}^{m} h\left(g\left(Y, e_{i}\right) X-g\left(X, e_{i}\right) Y, e_{i}\right) \\
& =2 c \sum_{i=1}^{m}\left\{g\left(Y, e_{i}\right) h\left(X, e_{i}\right)-g\left(X, e_{i}\right) h\left(Y, e_{i}\right)\right\}=2 c\{h(X, Y)-h(Y, X)\}=0,
\end{aligned}
$$

i.e.

$$
R^{\perp}(X, Y) H=0 .
$$

On the other hand, by (4.17) and (5.1),

$$
\begin{aligned}
0= & \left(R^{\widetilde{\nabla}}(X, Y) h\right)(Z, W)=R^{\perp}(X, Y) h(Z, W)-h(R(X, Y) Z, W)-h(Z, R(X, Y) W) \\
= & R^{\perp}(X, Y) J A_{J Z}^{*} W-J A_{J W}^{*} R(X, Y) Z-J A_{J Z}^{*} R(X, Y) W \\
= & c J\left\{g\left(Y, A_{J Z}^{*} W\right) X-g\left(X, A_{J Z}^{*} W\right) Y-g(Y, Z) A_{J W}^{*} X\right. \\
& \left.+g(X, Z) A_{J W}^{*} Y-g(Y, W) A_{J Z}^{*} X+g(X, W) A_{J Z}^{*} Y\right\} .
\end{aligned}
$$

Now we will put $X=W=e_{i}$ in (5.3) and add for $i=1, \ldots, m$, we get:

$$
\begin{aligned}
0 & =\sum_{i=1}^{m}\left\{g\left(A_{J Z}^{*} e_{i}, Y\right) e_{i}-g\left(A_{J Z}^{*} e_{i}, e_{i}\right) Y-g(Y, Z) A_{J e_{i}}^{*} e_{i}+g\left(e_{i}, Z\right) A_{J e_{i}}^{*} Y-g\left(Y, e_{i}\right) A_{J Z}^{*} e_{i}+g\left(e_{i}, e_{i}\right) A_{J Z}^{*} Y\right\} \\
& =\sum_{i=1}^{m}\left\{g\left(A_{J Z}^{*} Y, e_{i}\right) e_{i}-g\left(A_{J e_{i}}^{*} e_{i}, Z\right) Y-g(Y, Z) A_{J e_{i}}^{*} e_{i}+A_{g\left(e_{i}, Z\right) J e_{i}}^{*} Y-A_{J Z}^{*} g\left(Y, e_{i}\right) e_{i}+g\left(e_{i}, e_{i}\right) A_{J Z}^{*} Y\right\} \\
& =A_{J Z}^{*} Y-g(-m J H, Z) Y+g(Y, Z) m J H+A_{J Z}^{*} Y-A_{J Z}^{*} Y+m A_{J Z}^{*} Y \\
& =m g(J H, Z) Y+m g(Y, Z) J H+(m+1) A_{J Z}^{*} Y .
\end{aligned}
$$

From (5.2) and Proposition 5.3 it follows $H=0$, i.e. $A_{J Z}^{*} Y=0$, because of (5.4).

\section{Four Dimensional Holomorphic Statistical Manifolds}

In this section we will provide a method to construct holomorphic statistical structures on $\mathbb{R}^{4}$ using a $g$-natural metric $G$, defined by Oproiu in 1999 (see [5]).

It is known that the tangent bundle $T M$ of an $n$-dimensional Riemannian manifold $(M, g)$ has a structure of $2 n$-dimensional manifold induced from the manifold structure of $M$. A local chart $\left(U, x_{1}, \ldots, x_{n}\right)$ on $M$ induces a local chart $\left(\tau^{-1}(U), x_{1}, \ldots, x_{n}, y_{1}, \ldots, y_{n}\right)$ on $T M$, where $x_{i}=x_{i} \circ \tau, i=1, \ldots, n$, and $\tau: T M \rightarrow M$. The Levi-Civita connection of $g$ defines a direct sum decomposition $T T M=V T M \oplus H T M$ of the tangent bundle to TM into the vertical distribution VTM and the horizontal distribution HTM. The vector fields $\left(\frac{\partial}{\partial y_{1}}, \ldots, \frac{\partial}{\partial y_{n}}\right)$ and $\left(\frac{\delta}{\delta x_{1}}, \ldots, \frac{\delta}{\delta x_{n}}\right)$ define a local frame fields for VTM and HTM, respectively, where $\frac{\delta}{\delta x_{i}}=\frac{\partial}{\partial x_{i}}-\sum_{h=1}^{n} \Gamma_{i 0}^{h} \frac{\partial}{\partial y_{h}}, \Gamma_{i 0}^{h}=\sum_{k=1}^{n} \Gamma_{i k}^{h} y_{k}$ and $\Gamma_{i k}^{h}$ are the Christoffel symbols defined by $g$. Oproiu considered on $T M$ the energy density $t=\frac{1}{2} \sum_{i, k=1}^{n} g_{i k} y_{i} y_{k}$, where $g_{i k}$ are the components of $g$. The functions $u, v:[0, \infty) \rightarrow \mathbb{R}$ are chosen such that $u>0$ and $u+2 t v>0$. In this case, the $(0,2)$-tensor field $G_{i j}=u(t) g_{i j}+v(t) g_{0 i} g_{0 j}$, is considered, where $g_{0 i}=\sum_{h=1}^{n} g_{h i} y_{h}$. The inverse of the symmetric matrix $\left(G_{i j}\right)$ has the entries $H^{k l}=\frac{1}{u} g^{k l}+w y_{k} y_{l}$, where $g^{k l}$ are the components of the inverse of the matrix $\left(g_{i j}\right)$ and $w=w(t)=-\frac{v}{u(u+2 t v)}$. Denoting by $H_{i j}=\sum_{k, l=1}^{n} g_{i k} H^{k l} g_{l j}=\frac{1}{u} g_{i j}+w g_{0 i} g_{0 j}$, on $T M$ the Riemannian metric $G=\sum_{i, j=1}^{n}\left(G_{i j} d x_{i} d x_{j}+H_{i j} \nabla^{\prime} y_{i} \nabla^{\prime} y_{j}\right)$, is considered, where $\nabla^{\prime} y_{i}=d y_{i}+\sum_{j=1}^{n} \Gamma_{j 0}^{i} d x_{j}$. An almost complex structure $J$ on $T M$ is defined by $J \frac{\delta}{\delta x_{i}}=\sum_{k=1}^{n} G_{i}^{k} \frac{\partial}{\partial y_{k}}, J \frac{\partial}{\partial y_{i}}=-\sum_{k=1}^{n} H_{i}^{k} \frac{\delta}{\delta x_{k}}$, where $G_{i}^{k}=\sum_{h=1}^{n} G_{i h} g^{h k}$ and $H_{i}^{k}=\sum_{h=1}^{n} H_{i h} g^{h k}$. The Kähler form for $G$ and $J$ is defined by $\omega\left(.,{ }^{\circ}\right):=G(., J$.$) . We are using this construction for the manifold \left(\mathbb{R}^{2}, g\right)$, where $g$ is the Euclidean metric, in the following way.

Let $\left(x_{1}, x_{2}, y_{1}, y_{2}\right)$ be a standard coordinate system on $\mathbb{R}^{4}$. Take a function $a:[0, \infty) \rightarrow(0, \infty)$. Set $b(t):=$ $-a(t) a^{\prime}(t)\left(2 t a^{\prime}(t)-a(t)\right)^{-1}$ and assume $a(t)+2 t b(t)>0$ for $t \geq 0$. Define $t:=\left(y_{1}^{2}+y_{2}^{2}\right) / 2$ and functions $u, v$ on $\mathbb{R}^{4}$ by 
$u\left(x_{1}, x_{2}, y_{1}, y_{2}\right):=a(t), v\left(x_{1}, x_{2}, y_{1}, y_{2}\right):=b(t)$. Oproiu defined a $g$-natural metric $G$ on $\mathbb{R}^{4}$, and a complex structure $J$ by:

$$
\begin{aligned}
G= & \left(u+v y_{1}^{2}\right) d x_{1} d x_{1}+2 v y_{1} y_{2} d x_{1} d x_{2}+\left(u+v y_{2}^{2}\right) d x_{2} d x_{2}+\frac{u+v y_{2}^{2}}{u(u+2 t v)} d y_{1} d y_{1}-2 \frac{v y_{1} y_{2}}{u(u+2 t v)} d y_{1} d y_{2} \\
& +\frac{u+v y_{1}^{2}}{u(u+2 t v)} d y_{2} d y_{2}, \\
& J \frac{\partial}{\partial x_{1}}=\left(u+v y_{1}^{2}\right) \frac{\partial}{\partial y_{1}}+v y_{1} y_{2} \frac{\partial}{\partial y_{2}}, \quad J \frac{\partial}{\partial x_{2}}=v y_{1} y_{2} \frac{\partial}{\partial y_{1}}+\left(u+v y_{2}^{2}\right) \frac{\partial}{\partial y_{2}}, \\
& J \frac{\partial}{\partial y_{1}}=-\frac{u+v y_{2}^{2}}{u(u+2 t v)} \frac{\partial}{\partial x_{1}}+\frac{v y_{1} y_{2}}{u(u+2 t v)} \frac{\partial}{\partial x_{2}}, \quad J \frac{\partial}{\partial y_{2}}=\frac{v y_{1} y_{2}}{u(u+2 t v)} \frac{\partial}{\partial x_{1}}-\frac{u+v y_{1}^{2}}{u(u+2 t v)} \frac{\partial}{\partial x_{2}} .
\end{aligned}
$$

The metric $G$ and the complex structure $J$ are constructed so that $\mathbb{R}^{4}$ is Kählerian. For these metric $G$ and complex structure $J$ we construct a tensor field $K$ that satisfies the conditions of Lemma 4.2. That is, the following theorem holds.

Theorem 6.1. Let $G$ be a g-natural metric defined by (6.1) and $J$ a complex structure defined by (6.2). Then there exists a tensor field $K$ defined by nine functions on $\mathbb{R}^{4}$ such that $\bar{M}=\left(\mathbb{R}^{4}, \bar{\nabla}:=\nabla^{G}+K, G, J\right)$ is a holomorphic statistical manifold.

Proof. We calculate a $(1,2)$-tensor field $K=\sum_{i, j, l=1}^{4} k_{i j}^{l} \frac{\partial}{\partial x^{l}} \otimes d x^{i} \otimes d x^{j}$ on $\mathbb{R}^{4}$, in a way that it satisfies the conditions in Lemma 4.2. That is, $K$ satisfies (4.1), (4.4) and (4.5). For the convenience we denote by $p:=u+v y_{1}^{2}, q:=u+v y_{2}^{2}$, $r:=u+2 t v, s:=v y_{1} y_{2}$. We get the following.

$$
\begin{aligned}
& k_{14}^{1}=k_{41}^{1}=k_{13}^{2}=k_{31}^{2}=-k_{34}^{3}=-k_{43}^{3}=\alpha_{1}, k_{11}^{4}=k_{12}^{3}=k_{21}^{3}=\alpha_{2}, k_{12}^{4}=k_{21}^{4}=k_{22}^{3}=\alpha_{3} \text {, } \\
& k_{24}^{1}=k_{42}^{1}=k_{23}^{2}=k_{32}^{2}=-k_{34}^{4}=-k_{43}^{4}=\alpha_{4}, k_{22}^{2}=-k_{24}^{4}=-k_{42}^{4}=\alpha_{5} \text {, } \\
& k_{11}^{1}=k_{12}^{1}=k_{21}^{1}=-k_{23}^{3}=-k_{32}^{3}=\alpha_{6}, k_{12}^{2}=k_{21}^{2}=-k_{24}^{3}=-k_{42}^{3}=\alpha_{6} \frac{s-p}{s}+\alpha_{10} \frac{q}{s} \text {, } \\
& k_{33}^{1}=\alpha_{7}, k_{11}^{2}=\alpha_{8}, k_{14}^{3}=k_{41}^{3}=\alpha_{9}, k_{14}^{2}=k_{41}^{2}=-\alpha_{2} \frac{s}{u r q}+\alpha_{3} \frac{p}{u r q}-\alpha_{1} \frac{s}{q} \text {, } \\
& k_{23}^{1}=k_{32}^{1}=\alpha_{2} \frac{q}{u r p}-\alpha_{4} \frac{s}{p}-\alpha_{3} \frac{s}{u r p}, k_{11}^{3}=\alpha_{1} \frac{2 s^{4}-u^{2} r^{2}}{s q}+\alpha_{2} \frac{u r+2 s^{2}}{s q}-\alpha_{3} \frac{p}{q}, \\
& k_{22}^{4}=-\alpha_{2} \frac{q}{p}-\alpha_{4} \frac{u^{2} r^{2}}{s p}+\alpha_{3} \frac{u r+2 s^{2}}{s p}, k_{13}^{1}=k_{31}^{1}=\alpha_{2} \frac{q}{s u r}-\alpha_{3} \frac{1}{u r}-\alpha_{1} \frac{q}{s} \text {, } \\
& k_{33}^{4}=-\alpha_{2} \frac{q}{u r p}+\alpha_{3} \frac{s}{u r p}+\alpha_{4} \frac{s}{p}, k_{44}^{4}=-k_{24}^{2}=-k_{42}^{2}=\alpha_{2} \frac{1}{u r}-\alpha_{3} \frac{p}{s u r}+\alpha_{4} \frac{p}{s}, \\
& k_{44}^{3}=\alpha_{2} \frac{s}{q u r}-\alpha_{3} \frac{p}{q u r}+\alpha_{1} \frac{s}{q}, k_{33}^{3}=-\alpha_{2} \frac{q}{s u r}+\alpha_{3} \frac{1}{u r}+\alpha_{1} \frac{q}{s}, \\
& k_{22}^{1}=-k_{23}^{4}=-k_{32}^{4}=-\alpha_{5} \frac{s}{p}+\alpha_{6} \frac{s q-u r}{s p}+\alpha_{8} \frac{q^{2}}{s p}, \\
& k_{34}^{1}=k_{43}^{1}=-\alpha_{7} \frac{s}{q}+\alpha_{6} \frac{s q-2 u r-s^{2}}{u r q p}-\alpha_{5} \frac{s^{2}}{u r p q}+\alpha_{8} \frac{q}{u r p}, \\
& k_{44}^{1}=\alpha_{7} \frac{s^{2}}{q^{2}}+\alpha_{6}\left(\frac{s}{q^{2} p}-\frac{(s-p)\left(2 s^{2}+u r\right)}{s u r p q}\right)+\alpha_{5} \frac{s\left(2 s^{2}+u r\right)}{u r p q^{2}}-\alpha_{8} \frac{2 s^{2}+u r}{s u r p}, \\
& k_{13}^{3}=k_{31}^{3}=-\alpha_{8} \frac{s}{p}-\alpha_{6}-\alpha_{9} \frac{s}{p}, k_{14}^{4}=k_{41}^{4}=\alpha_{6} \frac{(p)-s}{s}+\alpha_{9} \frac{q}{s}, \\
& k_{13}^{4}=k_{31}^{4}=\alpha_{8} \frac{u r}{p^{2}}-\alpha_{6} \frac{u+r}{p}-\alpha_{9} \frac{s^{2}}{p^{2}}, k_{33}^{2}=-\alpha_{8} \frac{q}{p u r}-\alpha_{9} \frac{q}{u r p}+\alpha_{6} \frac{s-q}{u r}-\alpha_{7} \frac{p}{s} \text {, } \\
& k_{34}^{2}=k_{43}^{2}=-\alpha_{8} \frac{1}{p s}+\alpha_{9} \frac{s}{u r p}+\alpha_{7} \frac{p}{q}+\alpha_{5} \frac{s}{u r q}+\alpha_{6} \frac{1}{q s}, \\
& k_{44}^{2}=-\alpha_{5} \frac{p q+s^{2}}{u r q^{2}}+\alpha_{8} \frac{2 u r+s^{2}}{u r p q}-\alpha_{9} \frac{s^{2}}{u r p q}-\alpha_{7} \frac{s p}{q^{2}}+\alpha_{6}\left(-\frac{1}{q^{2}}+\frac{s-p}{u r q}\right) \text {, }
\end{aligned}
$$

where $\alpha_{1}, \ldots, \alpha_{9}$ are functions. Then a manifold $\bar{M}=\left(\mathbb{R}^{4}, \bar{\nabla}:=\nabla^{G}+K, G, J\right)$ is holomorphic statistical. To show this, we calculate the next, for $\frac{\partial}{\partial x_{1}}, \frac{\partial}{\partial x_{2}}, \frac{\partial}{\partial y_{1}} \in \Gamma(T \bar{M})$.

(1)

$$
\bar{\nabla}_{\frac{\partial}{\partial x_{1}}} \frac{\partial}{\partial y_{1}}=\nabla_{\frac{\partial}{\partial x_{1}}}^{G} \frac{\partial}{\partial y_{1}}+K\left(\frac{\partial}{\partial x_{1}}, \frac{\partial}{\partial y_{1}}\right)=\nabla_{\frac{\partial}{\partial x_{1}}}^{G} \frac{\partial}{\partial y_{1}}+k_{13}^{1} \frac{\partial}{\partial x_{1}}+k_{13}^{2} \frac{\partial}{\partial x_{2}}+k_{13}^{3} \frac{\partial}{\partial y_{1}}+k_{13}^{4} \frac{\partial}{\partial y_{2}},
$$


and

$$
\begin{aligned}
\bar{\nabla}_{\frac{\partial}{\partial y_{1}}} \frac{\partial}{\partial x_{1}} & =\nabla_{\frac{\partial}{\partial y_{1}}}^{G} \frac{\partial}{\partial x_{1}}+k_{31}^{1} \frac{\partial}{\partial x_{1}}+k_{31}^{2} \frac{\partial}{\partial x_{2}}+k_{31}^{3} \frac{\partial}{\partial y_{1}}+k_{31}^{4} \frac{\partial}{\partial y_{2}} \\
& =\nabla_{\frac{\partial}{\partial y_{1}}}^{G} \frac{\partial}{\partial x_{1}}+k_{13}^{1} \frac{\partial}{\partial x_{1}}+k_{13}^{2} \frac{\partial}{\partial x_{2}}+k_{13}^{3} \frac{\partial}{\partial y_{1}}+k_{13}^{4} \frac{\partial}{\partial y_{2}} .
\end{aligned}
$$

Subtracting the last two equations, we get

$$
\bar{\nabla}_{\frac{\partial}{\partial x_{1}}} \frac{\partial}{\partial y_{1}}-\bar{\nabla}_{\frac{\partial}{\partial y_{1}}} \frac{\partial}{\partial x_{1}}=\nabla_{\frac{\partial}{\partial x_{1}}}^{G} \frac{\partial}{\partial y_{1}}-\nabla_{\frac{\partial}{\partial y_{1}}}^{G} \frac{\partial}{\partial x_{1}}=\left[\frac{\partial}{\partial x_{1}}, \frac{\partial}{\partial y_{1}}\right]
$$

since $\nabla^{G}$ is of torsion free, as being a Levi-Civita connection.

(2)

$$
\begin{aligned}
\left(\bar{\nabla}_{\frac{\partial}{\partial x_{1}}} G\right)\left(\frac{\partial}{\partial x_{2}}, \frac{\partial}{\partial y_{1}}\right)= & G\left(\nabla_{\frac{\partial}{\partial x_{1}}}^{G} \frac{\partial}{\partial x_{2}}, \frac{\partial}{\partial y_{1}}\right)+G\left(\frac{\partial}{\partial x_{2}}, \nabla_{\frac{\partial}{\partial x_{1}}}^{G} \frac{\partial}{\partial y_{1}}\right)-G\left(\bar{\nabla}_{\frac{\partial}{\partial x_{1}}} \frac{\partial}{\partial x_{2}}, \frac{\partial}{\partial y_{1}}\right) \\
& -G\left(\frac{\partial}{\partial x_{2}}, \bar{\nabla}_{\frac{\partial}{\partial x_{1}}} \frac{\partial}{\partial y_{1}}\right)=-2 G\left(k_{12}^{1} \frac{\partial}{\partial x_{1}}+k_{12}^{2} \frac{\partial}{\partial x_{2}}+k_{12}^{3} \frac{\partial}{\partial y_{1}}+k_{12}^{4} \frac{\partial}{\partial y_{2}}, \frac{\partial}{\partial y_{1}}\right) \\
= & k_{12}^{3} \frac{u+v y_{2}^{2}}{u(u+2 t v)}-k_{12}^{4} \frac{v y_{1} y_{2}}{u(u+2 t v)},
\end{aligned}
$$

and

$$
\left(\bar{\nabla}_{\frac{\partial}{\partial x_{2}}} G\right)\left(\frac{\partial}{\partial x_{1}}, \frac{\partial}{\partial y_{1}}\right)=k_{21}^{3} \frac{u+v y_{2}^{2}}{u(u+2 t v)}-k_{21}^{4} \frac{v y_{1} y_{2}}{u(u+2 t v)}=k_{12}^{3} \frac{u+v y_{2}^{2}}{u(u+2 t v)}-k_{12}^{4} \frac{v y_{1} y_{2}}{u(u+2 t v)} .
$$

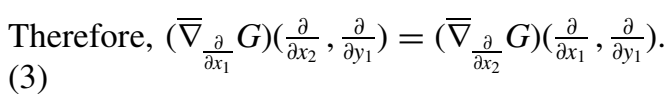

$$
\begin{aligned}
\left(\bar{\nabla}_{\frac{\partial}{\partial x_{1}}} \omega\right)\left(\frac{\partial}{\partial x_{2}}, \frac{\partial}{\partial y_{1}}\right)= & -G\left(K\left(\frac{\partial}{\partial x_{1}}, \frac{\partial}{\partial x_{2}}\right), J \frac{\partial}{\partial y_{1}}\right)+G\left(\frac{\partial}{\partial x_{2}}, K\left(\frac{\partial}{\partial x_{1}}, J \frac{\partial}{\partial y_{1}}\right)\right) \\
& -G\left(K\left(\frac{\partial}{\partial x_{1}}, \frac{\partial}{\partial x_{2}}\right), J \frac{\partial}{\partial y_{1}}\right)+G\left(K\left(\frac{\partial}{\partial x_{1}}, \frac{\partial}{\partial x_{2}}\right), J \frac{\partial}{\partial y_{1}}\right) \\
= & -\frac{q}{u r} s\left(-\alpha_{8} \frac{q}{s}+\alpha_{6} \frac{p}{s}+\alpha_{6} \frac{s-p}{s}+\alpha_{8} \frac{q}{s}\right)-\alpha_{8} \frac{q^{2}}{u r}+\alpha_{6} \frac{s^{2}}{u r}+\left(\alpha_{6} \frac{s-p}{s}+\alpha_{8} \frac{q}{s}\right) \frac{s}{u r} q \\
& +\frac{q}{u r} p k_{11}^{1}-\frac{s^{2}}{u r} k_{11}^{1}=-\alpha_{6}+k_{11}^{1}=0 .
\end{aligned}
$$

Therefore, the conditions in Definition 4.1 are satisfied for the chosen basis elements of $\Gamma(T \bar{M})$.

Let the function $u$ in the proof of Theorem 6.1 be defined as $u:=\frac{1+\sqrt{1+4 t}}{2}$ and $p, q, r, s$ be defined in the same way. And let

$$
\alpha_{6}=\alpha_{8}=\alpha_{9}=0, \quad \alpha_{2}=\frac{1}{2} s\left(u_{y_{1}}+2 y_{1}\right)+\frac{1}{2} q u_{y_{2}},
$$

and assume that $\alpha_{1}$ and $\alpha_{3}$ satisfy the following equation:

$$
\left(\alpha_{2} \frac{q}{s u r}-\alpha_{3} \frac{1}{u r}-\alpha_{1} \frac{q}{s}\right) \frac{p u r}{q}+\alpha_{1} \frac{s u r}{q}+\alpha_{2} \frac{s}{q}=\frac{1}{2} p\left(u_{y_{1}}+2 y_{1}\right)+\frac{1}{2} s u_{y_{2}},
$$

where $u_{y_{1}}:=\frac{\partial u}{\partial y_{1}}$ and $u_{y_{2}}:=\frac{\partial u}{\partial y_{2}}$.

Corollary 6.2. Let $K_{0}$ be a tensor field constructed as in the proof of Theorem 6.1. If $K_{0}$ also satisfies the conditions (6.3) and (6.4) then $\bar{M}=\left(\mathbb{R}^{4}, \bar{\nabla}:=\nabla^{G}+K_{0}, G, J\right)$ is a special Kähler manifold.

Sketch of proof. For $\frac{\partial}{\partial_{x_{1}}}, \frac{\partial}{x_{x_{2}}}, \frac{\partial}{\partial_{y_{1}}} \in \Gamma(T \bar{M})$, we calculate that $\bar{R}\left(\frac{\partial}{\partial_{x_{1}}}, \frac{\partial}{\partial_{x_{2}}}\right) \frac{\partial}{\partial_{y_{1}}}=0$. On all the others combinations of the basis elements we get that the curvature tensor $\bar{R}$ vanishes.

Motivated by Theorem 1.1 and Theorem 4.9, here we give examples of two dimensional submanifolds $M$ of holomorphic statistical manifolds defined in Theorem 6.1 which satisfy the condition

$$
I(X, Y) \in \Gamma\left(T^{\perp} M\right), X, Y \in \Gamma(T M) .
$$

Example 6.3. Let $\bar{M}$ be the holomorphic statistical manifold in Theorem 6.1 with functions $\alpha_{1}, \ldots, \alpha_{9}$ satisfying $\alpha_{1}=\alpha_{2}=\alpha_{3}=\alpha_{4}=0$ and $\alpha_{8}$ is a function of variables $y_{1}$ and $y_{2}$. Let $M$ be a submanifold of $\bar{M}$ such that $A_{1}=\frac{\partial}{\partial x_{1}}$ is a 
unitary vector field tangent to $M$. In this case $A_{2}=\alpha_{8} \frac{s}{p} \frac{\partial}{\partial x_{1}}-\alpha_{8} \frac{\partial}{\partial x_{2}}$ is a unitary vector field orthogonal to $A_{1}$ and tangent to $M$. Calculating the Lie bracket of $A_{1}$ and $A_{2}$ we get $\left[A_{1}, A_{2}\right]=0$, therefore a submanifold $M$ tangent to $A_{1}$ and $A_{2}$ exists.

Consider now that $M$ is given by

$$
\begin{aligned}
& f_{1}\left(x_{1}, x_{2}, y_{1}, y_{2}\right)=0, \\
& f_{2}\left(x_{1}, x_{2}, y_{1}, y_{2}\right)=0,
\end{aligned}
$$

where $f_{1}$ and $f_{2}$ are smooth functions on $\mathbb{R}^{4}$ satisfying rank $\frac{D\left(f_{1}, f_{2}\right)}{D\left(x_{1}, x_{2}, y_{1}, y_{2}\right)}=2$. Since $A_{1}$ and $A_{2}$ are tangent to $M$ we have

$$
\begin{aligned}
& \frac{\partial f_{i}}{\partial x_{1}}=0, \\
& \alpha_{8} \frac{s}{p} \frac{\partial f_{i}}{\partial x_{1}}-\alpha_{8} \frac{\partial f_{i}}{\partial x_{2}}=0, \quad i=1,2 .
\end{aligned}
$$

Then it follows $\frac{\partial f_{i}}{\partial x_{2}}=0$, hence $f_{i}=f_{i}\left(y_{1}, y_{2}\right), i=1,2$. Consequently, $M$ is given by $y_{1}=$ constant and $y_{2}=$ constant, that is, $M$ is a portion of a 2 -plane.

We remark here that in this case the condition $I(X, Y) \in T^{\perp} M$ is satisfied for $X, Y \in \Gamma(T M)$. Also, vectors

$$
\begin{aligned}
& B_{1}=\alpha_{8} \frac{u r}{p} \frac{\partial}{\partial y_{2}}, \\
& B_{2}=p \frac{\partial}{\partial y_{1}}+s \frac{\partial}{\partial y_{2}},
\end{aligned}
$$

are unitary, orthogonal and generate the normal bundle of $M$. We can calculate the mean curvature vector field as

$$
\begin{aligned}
H= & \left\{-\frac{1}{2 \alpha_{8}} u_{y_{2}}+\alpha_{8}\left(-\frac{1}{2} \frac{s^{2}}{p^{2}} u_{y_{2}}+\frac{y_{1} s}{p}-\frac{1}{2} u_{y_{2}}-y_{2}\right)\right\} B_{1} \\
& +\left\{\frac{1}{2}\left(-u_{y_{1}}-2 y_{1}\right)-\frac{1}{2} u_{y_{2}} \frac{s}{p}+\frac{\alpha_{8}^{2}}{2} \frac{s^{2}}{p^{2}}\left(-u_{y_{1}}-2 y_{1}\right)-\frac{\alpha_{8}^{2}}{2} \frac{s^{3}}{p^{3}} u_{y_{2}}+\frac{\alpha_{8}^{2} s}{p^{2}}\left(p y_{2}+y_{1} s\right)\right. \\
& \left.-\frac{\alpha_{8}^{2}}{2 p}\left(p u_{y_{1}}+s\left(u_{y_{2}}+2 y_{2}\right)\right)\right\} B_{2} .
\end{aligned}
$$

\section{REFERENCES}

[1] Abbassi, M. T. K., and Sarih, M., "On some hereditary properties of Riemannian g-natural metrics on tangent bundles of Riemannian manifolds,” Diff. Geom. Appl., 22: 19-47 (2005).

[2] Alekseevsky, D. V., Cortes, V., and Devchand, C., "Special complex manifolds," J. Geom. Phys., 42: 85-105 (2002).

[3] Furuhata, H., "Hypersurfaces in statistical manifolds," Diff. Geom. Appl., 27: 420-429 (2009).

[4] Kassabov, O., "On totally real submanifolds," Bull. Soc. Math. Belg., Sér. B, 38(2): 136-143 (1986).

[5] Oproiu, V., "Some new geometric structures on the tangent bundle," Publ. Math. Debrecen, 55: 261-281 (1999).

[6] Vos, P. W., "Fundamental equations for statistical submanifolds with applications to the Bartlett correction," Ann. Inst. Statist. Math., 41(3): 429-450 (1989).

[7] Yano, K., and Kon, M., “Totally real submanifolds of complex space forms, I,” Tohoku Math. J. (2), 2(2): $215-225$ (1976).

[8] Yano, K., and Kon, M., CR Submanifolds of Kaehlerian and Sasakian Manifolds, Birkhäuser (1983). 